\title{
A New Bound for the Gamma FunctionIn the Direction of W.K.Hayman
}

\author{
K.S.L.N.Prasad \\ Associate professor,Dept.of Mathematics,Karnatak Arts College, Dharwad-580001.
}

Abstract: In this paper I have extended a result of W.K.Hayman to Euler's gamma function which is known to be a logarithmically Convex function.

Key Words: Euler's gamma function, convex function.

\section{Introduction And Main Results}

By Bohr - Mollerup- Artin theorem, we can define the Euler gamma function as follows.

Theorem A: Let $\mathrm{f}: \mathrm{R}^{+} \rightarrow \mathrm{R}^{+}$satisfy each of the following properties.

i) $\quad \log f(x)$ is a convex function

ii) $\quad \mathrm{f}(\mathrm{x}+1)=\mathrm{xf}(\mathrm{x}) \quad \forall \mathrm{x} \in \mathrm{R}^{+}$

iii) $\quad \mathrm{f}(1)=1$

Then, $\mathrm{f}(\mathrm{x})=\Gamma(\mathrm{x}), \forall \mathrm{x} \in \mathrm{R}^{+}$

But,Usually the Euler Gamma function is introduced as a function of a real variable and is defined via an integral

$$
\Gamma(\mathrm{x})=\int_{0}^{\infty} \mathrm{e}^{-\mathrm{t}} \mathrm{t}^{\mathrm{x}-1} \mathrm{dt}, \mathrm{x}>0
$$

Here, we can observe that $\Gamma(\mathrm{x}+1)=\mathrm{x}$ !

One can easily verify the following properties.

Property 1 :

\section{$\Gamma(\mathrm{x})$ is a logarithmatically convex function}

(or)

$$
f(x)=\log \Gamma(x) \text { is a convex function. }
$$

The proof of this property follows directly from the following definition.

\section{Definition :}

Let $\Phi(\mathrm{x})$ be a real valued function on $[\mathrm{a}, \mathrm{b}]$ and let

$\Phi^{\|}(\mathrm{x}) \geq 0$ for all $\mathrm{x} \in[\mathrm{a}, \mathrm{b}]$. Or, Equivalently,Let $\Phi^{\mid}$be non decreasing on $[\mathrm{a}, \mathrm{b}]$.Then, $\Phi$ is a convex function on $[\mathrm{a}, \mathrm{b}]$.

Or $\Phi$ is said to be convex on $[a, b]$ iff

$$
\left|\begin{array}{lll}
\Phi\left(\mathrm{x}_{1}\right) & \mathrm{x}_{1} & 1 \\
\Phi\left(\mathrm{x}_{2}\right) & \mathrm{x}_{2} & 1 \\
\Phi\left(\mathrm{x}_{3}\right) & \mathrm{x}_{3} & 1
\end{array}\right| \leq 0
$$

i.e. $\Phi\left(\mathrm{x}_{1}\right)\left(\mathrm{x}_{3}-\mathrm{x}_{2}\right)+\Phi\left(\mathrm{x}_{3}\right)\left(\mathrm{x}_{2}-\mathrm{x}_{1}\right) \geq \Phi\left(\mathrm{x}_{2}\right)\left(\mathrm{x}_{3}-\mathrm{x}_{1}\right)---\rightarrow(1)$

Clearly, one can observe that $\Phi(\mathrm{x})=\log \Gamma(\mathrm{x})$ statisfies (1) and hence $\log \Gamma(\mathrm{x})$ is a convex function on [a, b].

We wish to establish the following theorem.

Theorem :

Suppose that $\mathbf{g}(\mathbf{x})=\log \Gamma(\mathrm{x})$ is a strictly increasing and convex

function of $x$ for $x \geq x_{0}$. Then given $k>1$ there exists a sequence

$x_{n} \rightarrow \infty$ such that If $f(x)$ is any other positive increasing and convex

function of $x$ such that $f(x)<g(x)$ for $x \geq x_{0}$, then we have,

$$
\Gamma\left(x_{n}\right) f^{\prime}\left(x_{n}\right)<e^{k} \Gamma^{\prime}\left(x_{n}\right) \quad(n=1,2, \ldots . .)
$$


Here, $f^{\prime}(x)$ denotes the right derivative of $f(x)$ and $\frac{\Gamma^{\mid}(x)}{\Gamma\left(x_{n}\right)}$ is the left derivative of $g(x)$.

To prove the above result, we require the following lemma [Hayman].

Lemma[1] : Suppose that $\Phi(\mathrm{x})$ is positive for $\mathrm{x} \geq \mathrm{x}_{0}$ and bounded in every interval $\left[\mathrm{x}_{0}, \mathrm{x}_{1}\right]$ when $\mathrm{x}_{0}<\mathrm{x}_{1}<\infty$. Then given $\mathrm{k}>1$ there exists a sequence $\mathrm{x}_{\mathrm{n}} \rightarrow \infty$ such that

$\Phi(\mathrm{x})<\mathrm{k} \Phi\left(\mathrm{x}_{\mathrm{n}}\right)$ for $\mathrm{x}_{\mathrm{n}}<\mathrm{x}<\mathrm{x}_{\mathrm{n}}+\frac{1}{\log ^{+}\left[\Phi\left(\mathrm{x}_{\mathrm{n}}\right)\right]^{\mathrm{k}}}+\frac{1}{\Phi\left(\mathrm{x}_{\mathrm{n}}\right)}$

\section{Proof of the theorem :}

Since $\mathrm{g}(\mathrm{x})=\log \Gamma(\mathrm{x})$ is convex, $\mathrm{g}(\mathrm{x})$ is non decreasing. Since $\mathrm{g}(\mathrm{x})$ is strictly increasing, $\mathrm{g}^{\prime}(\mathrm{x})>0$ for $\mathrm{x}>\mathrm{x}_{0}$. Also $\mathrm{g}(\mathrm{x})$ is bounded above in any finite

interval $\left(\mathrm{x}_{0}, \mathrm{x}_{1}\right)$ for $\mathrm{x}_{1}>\mathrm{x}_{0}$. Thus, we may apply the above lemma to the function $\Phi(\mathrm{x})=\frac{\mathrm{g}(\mathrm{x})}{\mathrm{g}(\mathrm{x})}$ and hence we can find a sequence $\mathrm{x}_{\mathrm{n}}->\infty$ such that.

$\Phi(\mathrm{x})<\mathrm{k} \Phi\left(\mathrm{x}_{\mathrm{n}}\right)$ for $\mathrm{x}_{\mathrm{n}} \leq \mathrm{x} \leq \mathrm{x}_{\mathrm{n}}+\frac{1}{\Phi\left(\mathrm{x}_{\mathrm{n}}\right)}$

Also, if $x_{n}^{\prime}=x_{n}+\frac{1}{\Phi\left(x_{n}\right)}$,We have,

$$
\log g\left(x_{n}^{\mid}\right)-\log g\left(x_{n}\right)=\int_{x_{n}}^{x_{n}} \frac{g(x)}{g(x)} d x<\left(x_{n}^{\mid}-x_{n}\right) k \Phi\left(x_{n}\right)=k .
$$

Hence, $g\left(x_{n}^{\dagger}\right)<e^{k} g\left(x_{n}\right)$

Then, Since $f(x)$ is increasing, we have

$$
\begin{aligned}
f\left(x_{n}\right) \leq & \frac{1}{x_{n}^{\rfloor}-x_{n}} \int_{x_{n}}^{x_{n}^{\dagger}} f^{\prime}(x) d x=\frac{f\left(x_{n}^{\rfloor}\right)-f\left(x_{n}\right)}{x_{n}^{\backslash}-x_{n}} \leq \Phi\left(x_{n}\right) f\left(x_{n}^{\rfloor}\right) \\
& \leq \Phi\left(x_{n}\right) g\left(x_{n}^{\rfloor}\right) \\
& \leq e^{k} \Phi\left(x_{n}\right) g\left(x_{n}\right) \\
& =e^{k} g\left(x_{n}\right)
\end{aligned}
$$

Thus, $\mathrm{f}\left(\mathrm{x}_{\mathrm{n}}\right) \leq \mathrm{e}^{\mathrm{k}} \mathrm{g}\left(\mathrm{x}_{\mathrm{n}}\right)$

Or $\quad f\left(x_{n}\right) \leq e^{k} \frac{\Gamma^{\mid}\left(x_{n}\right)}{\Gamma\left(x_{n}\right)}$

Or $\quad \Gamma\left(\mathrm{x}_{\mathrm{n}}\right) \mathrm{f}^{\prime}\left(\mathrm{x}_{\mathrm{n}}\right)<\mathrm{e}^{\mathrm{k}} \Gamma^{\mid}\left(\mathrm{x}_{\mathrm{n}}\right)(\mathrm{n}=1,2,3 \ldots \ldots)$

\section{References}

[1]. HAYMAN W.K (1964):Meromorphic functions, Oxford Univ, Press, London.

[2]. YANG LO,(1982): Value distribution theory,Science press,Beijing, 